\title{
La Antigüedad Grecolatina en perspectiva interdisciplinaria
}

\section{(4) Julieta Cardigni \\ Universidad de Buenos Aires/CONICET, Argentina \\ jcardigni@yahoo.es}

\author{
Rodrigo Laham Cohen \\ Universidad de Buenos Aires/CONICET/Universidad Nacional de San Martín, \\ Argentina \\ R_lahamcohen@hotmail.com
}

\author{
Esteban Noce \\ Universidad de Buenos Aires/CONICET, Argentina \\ esteban.noce@filo.uba.ar
}

\section{Diego Paiaro}

Universidad de Buenos Aires/CONICET/Universidad Nacional de General Sarmiento, Argentina

diegopaiaro@hotmail.com

\author{
Mariano Requena \\ Universidad de Buenos Aires/Universidad Nacional de General Sarmiento/ \\ Universidad Nacional de San Martín, Argentina \\ marianorequena@gmail.com
}

\section{Analía Sapere \\ Universidad de Buenos Aires/CONICET, Argentina analiasapere@gmail.com}

En el presente volumen se compilan los trabajos que formaron parte del Primer Workshop Interdisciplinario de la Antigüedad Grecolatina, realizado los días 4 y 5 de agosto de 2017 en la Facultad de Filosofía y Letras de la Universidad de Buenos Aires. El espacio fue creado con el objetivo de fomentar el diálogo entre especialistas de distintas disciplinas que abordan temáticas vinculadas con la Antigüedad Clásica, intentando que en dicho intercambio se produzca un genuino cruce de miradas y metodologías. Con el propósito de encauzar la 
discusión, el Workshop planteó dos ejes específicos de análisis, que se llevaron a cabo en dos encuentros: por un lado, debates religiosos en la Antigüedad; por otro, reflexiones sobre política y sociedad. Tanto la organización del Workshop como la coordinación de ambas instancias estuvieron a cargo de Julieta Cardigni, Rodrigo Laham Cohen, Esteban Noce, Diego Paiaro, Mariano Requena y Analía Sapere, docentes e investigadores de la Facultad de Filosofía y Letras de la UBA.

En el marco del primer eje, contamos con trabajos sobre judaísmo y cristianismo que, partiendo de un exhaustivo trabajo con fuentes, dan cuenta de las implicancias sociales y políticas de las problemáticas en cuestión. Rodrigo Laham Cohen (UBA/UNSAM/CONICET) examina las referencias a Jesús halladas en el Talmud Yerushalmi a fin de exhibir de qué modo operan como marcas de la imposición de la autoridad rabínica sobre manifestaciones religiosas tanto judías como no judías. Paola Druille (UNLPam/CONICET) ofrece una indagación acerca de la gerousía en Alejandría ptolemaica a partir de un minucioso estudio filológico de documentación epigráfica. El artículo rescata el valor de la inscripción SGE 34.1532 para la reconstrucción de las instituciones alejandrinas del siglo II a. C. Mariano Spléndido (UNLP/CONICET) analiza el episodio de Ananías y Safira en Hechos de los Apóstoles (5.1-11), para desentrañar su sentido en el esquema general de la obra, que puede interpretarse en el marco de una reflexión sobre el liderazgo carismático e itinerante. Esteban Noce (UBA/CONICET) centra su trabajo en el estudio del sermón XLVIII de Máximo de Turín, para determinar la distinción semántica de los términos gentilitas y paganismus, y sus implicancias para un mejor conocimiento acerca de la presencia del cristianismo en el Piamonte de la época.

En cuanto al eje sobre política y sociedad, la discusión se traslada a Grecia antigua. María Cecilia Colombani (UM/UNMDP/UBACyT) explora la construcción del liderazgo del rey en la Teogonía de Hesíodo, en el marco de un abordaje que considera dicha obra como un esbozo filosófico imbricado en el mito. El artículo de Analía Sapere (UBA/CONICET) realiza un análisis textual de las biografías atenienses de Plutarco para dar cuenta de la forma en que, de acuerdo con el planteo del autor, los políticos se valen de estrategias teatrales para consolidar sus liderazgos. Finalmente, Marisa Divenosa (UBA/UNLa) recorre las cartas y fragmentos de Epicuro para reflexionar acerca de la tensión que allí se evidencia entre la postura apolítica del autor y su valoración positiva de la philía. Hallamos en estos textos un recorrido que contempla diferentes períodos de Grecia antigua, lo que permite exhibir y poner en paralelo distintas consideraciones respecto de la construcción de poder, la política y la sociedad, sobre la base de las herramientas que proveen la filología, la historia y la filosofía.

Esperamos a partir de la presente compilación contribuir con las discusiones en torno a la Antigüedad Grecolatina, enfatizando una vez más nuestra elección metodológica sustentada en la interdisciplina, dado que la complejidad del objeto de estudio, según se desprende de los temas tratados en los artículos, requiere sin lugar a dudas de un enfoque amplio y transversal. 